\title{
Mal-absorptive bariatric procedure causing keshan's disease
}

\begin{abstract}
Aims and objectives: The aim of this case study is to emphasize the importance of understanding and managing nutrition in planning and execution of malabsorptive bariatric surgeries.

Case summary: A 51-year-old female came with excessive weight loss and cardiomyopathy one year after undergoing laparoscopic mini gastric bypass at another hospital. She had no comorbidities before the surgery. Post surgery, she developed excessive weight loss and congestive cardiac failure. On further investigations, she was found to have severe nutritional deficiencies including that of selenium, leading to Keshan's disease. After optimization, a reversal of the mini gastric bypass was planned. Post surgery, patient was discharged on Post Operative Day seven. Six months after the surgery her weight increased by $25 \mathrm{~kg}$, and she is nutritionally well maintained with normal cardiac function.
\end{abstract}

Conclusion: Nutritional deficiencies are common post bariatric surgery, and micronutrient deficiencies may be missed. It is important to add nutritional supplements, maintain a close follow up, and to take the appropriate corrective measures in time.

Keywords: bariatric surgery, mini gastric bypass, malabsorption, selenium deficiency, keshan's disease, robotic surgery
Volume 6 Issue I - 2017

\author{
Palep JH,' Khandelwal NK, ${ }^{2}$ Kahale $\mathrm{P}^{3}$ Singhal \\ $\mathrm{T}^{4}$ \\ 1,2Department of Bariatric Surgery, Kokilaben Dhirubhai Ambani \\ Hospital, India \\ ${ }^{3}$ Department of Cardiology, Kokilaben Dhirubhai Ambani \\ Hospital, India \\ ${ }^{4}$ Department of infectious Diseases, Kokilaben Dhirubhai \\ Ambani Hospital, India
}

\section{Correspondence: Khandelwal Nidhi K, Senior Clinical Assistant, Department of Bariatric Surgery Kokilaben Dhirubhai Ambani Hospital, Mumbai 400053, Maharashtra, India, Tel +919819154755, Email nidhikkhandelwal@gmail.com}

Received: September 28, 2016 | Published: January 03, 2017
Abbreviations: EWL, excess weight loss; BMI, body mass index; URTI, upper respiratory tract infection; UTI, urinary tract infection; LVEF, left ventricular ejection fraction; CT, computed tomography; DJ, duodeno-jejunal; POD, post operative day; HRCT, high resolution computed tomography; BAL, bronchio-alveolar lavage; AKT, anti-koch's therapy; TPN, total parenteral nutrition; GI, gastrointestinal; $\mathrm{WHO}$, world health organization

\section{Introduction}

Bariatric surgery has become popular rapidly, not only to lose excess weight, but also to reverse and even prevent the onset of metabolic diseases, especially Type II Diabetes Mellitus. It is this beneficial metabolic effect that prompts patients to seek surgical management.

Multiple procedures have been described, with a variable extent of excess weight loss. The bypass procedures like Mini Gastric Bypass (MGB) and Roux-en-Y Gastric Bypass (RYGB) have higher percentage of Excess Weight Loss (EWL) (70-80\%) as compared to restrictive procedures like Sleeve Gastrectomy (SG) (50-60\%). One of the side effects of bypass procedures is excess weight loss along with severe malnutrition. The most common deficiencies are of iron, Vitamin B12 and Vitamin D3. However, deficiencies of trace elements are usually under - recognized. Laboratory testing for these is not routinely done, and most patients are unaware of their symptoms. Here we would like to report a rare case of Keshan's disease caused by excessive malabsorption post MGB.

\section{Case presentation}

A 51-year-old female patient came to us with complaints of excess weight loss, recurrent fever, poor appetite and anasarca. One year prior to seeing us she had undergone a Laparoscopic MGB at another hospital. Her weight before the first surgery was $85 \mathrm{~kg}$ and Body Mass Index (BMI) was $35 \mathrm{~kg} / \mathrm{m}^{2}$. She had no medical comorbidities to begin with. Post surgery, she was not started on any nutritional supplements by the primary surgeon. She gradually started losing weight and was comfortable and happy for the first four months. By the fifth month, she developed multiple episodes of loose stools, nausea and fever, for which she was hospitalized repeatedly. She soon started getting repeated episodes of URTIs and UTIs, all associated with high-grade fever, and each time she was given high-grade injectable antibiotics.

Table I Establishing the diagnosis of Keshan's disease

\begin{tabular}{lll}
\hline Test & Normal range & Patient's values on admission \\
\hline Selenium levels & $74-90 \mathrm{mcg} / \mathrm{l}$ & $47 \mathrm{mcg} / \mathrm{l}$ \\
BMI & $18.5-23 \mathrm{~kg} / \mathrm{m}^{2}$ & $15 \mathrm{~kg} / \mathrm{m}^{2}$ \\
LVEF & $>50 \%$ & $10-15 \%$ \\
\hline
\end{tabular}

She was losing excessive weight and her appetite dropped. She also had excessive hair fall, pallor, pedal edema, and skin excoriations all over. Laboratory tests showed severe anaemia with severe nutritional deficiencies of proteins and vitamins. Tests to rule out autoimmune diseases were also done. However, no tests for trace minerals were conducted. By 11 months post surgery, she developed anasarca with bilateral pleural effusions and ascites, complete loss of appetite, oral thrush and her weight dropped to $38 \mathrm{~kg}$ (total $47 \mathrm{~kg}$ loss since surgery) and BMI dropped to $15 \mathrm{~kg} / \mathrm{m}^{2}$. She soon developed breathlessness with hypoxia, and was diagnosed with congestive cardiac failure, and treated symptomatically with multiple cardiac medications. 2D echocardiogram showed a Left Ventricular Ejection 
Fraction (LVEF) of 10-15\% with dilated cardiomyopathy, mild Pulmonary Arterial hypertension and pericardial effusion. Ultrasound of the abdomen revealed congestive hepatomegaly with ascites. Once she was symptomatically better, she was transferred to our institute under the care of our cardiologist, who referred her to our Bariatric Surgery unit. All her laboratory investigations were repeated, and in addition tests for heavy elements and trace minerals were done. Our infectious disease specialist and pulmonologist were also involved. Computed Tomography (CT) guided pleural tapping was done, which helped decrease her dyspnea. Ultrasound of abdomen revealed sludge in the gall bladder. Her selenium levels were very low $(47 \mathrm{mcg} / \mathrm{l})$, thus establishing the diagnosis of Keshan's disease, and she was started on injectable selenium supplementation. Three days later, her selenium levels were within normal range $(86 \mathrm{mcg} / \mathrm{l})$, and on repeat echocardiography her LVEF showed a marked improvement, going up to $35 \%$

We planned a surgical reversal of the MGB with cholecystectomy. Four robotic ports (including the optical port) and one assistant port were used. Intra operatively, multiple dense adhesions were noted between the bowel loops and anterior abdominal wall. Also, after separating most adhesions, it was found that the transverse colon was densely adherent to the posterior wall of the gastroenterostomy. After separating the colon away, we found that the gastroenterostomy was not an anastomosis between the stomach and jejunum, but rather it was a gastroileostomy, $400 \mathrm{~cm}$ distal to the Duodeno-Jejunal (DJ) flexure, leaving only $50 \mathrm{~cm}$ of common channel of the ileum behind for absorption. This we believe was the reason behind her severe malnutrition, weight loss, and subsequent cardiomyopathy. We then disconnected the gastroileostomy, and the ileal margins were freshened and sutured with 3-0 PDS interrupted sutures. Following this, a robot assisted hand sewn gastro-gastrostomy was created between the two pouches of the stomach to re-establish intestinal continuity, using 3-0 PDS interrupted sutures.

Post surgery, feeds were started on POD two and gradually stepped up. She was discharged on POD six with reduced number of cardiac drugs. Rest of the course was uneventful, and the wound healed well. The patient's weight increased by two kg two weeks from the date of surgery, and was sent home on a high protein high calorie diet, with nutritional supplements.

However, three weeks post surgery, the patient developed lowgrade fever, cough with expectoration, and no further weight regain. Ultrasound of abdomen revealed no significant findings, and Chest $\mathrm{X}$ Ray was suggestive of recurrent left sided moderate pleural effusion. She was re-hospitalized, and a CT chest was done which was suggestive of Endobronchial Tuberculosis. A bronchio-alveolar lavage (BAL) sample and CT guided Supraclavicular Lymph Node Biopsy was done for a tissue diagnosis, which confirmed the presence of Mycobacterium Tuberculosis. The patient was re-referred to our Infectious Diseases Specialist, who started her on Anti-Koch's Therapy (AKT)

On her three-month follow up from the date of surgery, the patient had completed twomonths of the intensive phase of AKT, and was symptomatically better. In addition, she had put on $13 \mathrm{~kg}$, increasing her weight to $51 \mathrm{~kg}$ and BMI to $21.2 \mathrm{~kg} / \mathrm{m}^{2}$. Nutritionally, all her laboratory reports were within normal limits, and her quality of life was better. Her appetite had improved, there were no signs of pallor or anasarca as before, and her Echocardiogram showed further improvement in LV Ejection Fraction (55\%). All her cardiac drugs have been stopped.

\section{Discussion}

Keshan's disease was initially reported as a fatal disease endemic to China, and since then has been a rare medical entity all over the globe. ${ }^{1}$ It has been most conclusively associated with severe selenium deficiency. It affects the myocardium, causing myocyte loss and fibrous replacement. ${ }^{2}$ Rapid improvement is usually seen with supplementation of selenium. In addition to geographic influences, chronic malnutrition, prolonged Total Parenteral Nutrition use and Gastro-Intestinal disorders are etiological factors causing selenium deficiency. ${ }^{2}$

Bariatric surgery, especially malabsorptive procedures, has been criticized as leading to prolonged macro- and micronutrient deficiencies. ${ }^{3-5}$ Deficiencies of Vitamin B12, Iron, Vitamin D3 and Folate are very common, and usually manifest with anaemia, lethargy, and even pathological fractures. ${ }^{6,7}$ It is recommended to document their baselines values and monitor at frequent intervals post surgery, along with mandatory supplementation. Deficiencies of Vitamin $\mathrm{A}, \mathrm{C}, \mathrm{E}$ and $\mathrm{K}$ have also been documented, and most multivitamin supplements with an adequate diet can avoid overt deficiencies. ${ }^{6,7}$ Micronutrient deficiencies of trace elements like Copper, Zinc, Selenium, Molybdenum, Manganese and Chromium are very commonly missed..$^{4-8}$ Symptoms of these are usually attributed to a lack of Iron, B12 or Folate. Recalcitrant anaemia, excessive hair fall, skin changes, liver insufficiency, and cardiac failure are the warning signs. At our institute, we monitor these values at month's one, three, six, twelve, eighteen and twenty-four post surgery. In case of any abnormalities, supplements are adjusted accordingly.

In patients presenting with lethargy, refractory anaemia, diarrhea, recurrent infections and cardiac failure, selenium deficiency should be ruled out. ${ }^{1,2}$ In addition to measuring serum selenium levels, glutathione peroxidase activity can also be assessed, since selenium is an essential cofactor for this enzyme. ${ }^{2}$ Selenium can be replaced intravenously, and improvement in cardiac status is usually noted within a few days. The World Health Organization recommends a daily intake of $30-50 \mathrm{mcg} /$ day of selenium, but requirements after Bariatric Surgery are higher, and not clearly defined. Patients with heart failure need at least twice the daily recommended intake. ${ }^{2}$

Only two reports of severe Selenium deficiency have been documented post Bariatric Surgery to our knowledge. ${ }^{2,9,10}$ In both cases, the patient recovered well with adequate supplementation. However, in our case, since the patient was severely under nourished with excessive weight loss hampering her quality of life, we decided to reverse the original surgery.

\section{Conclusion}

Trace elements are deficient in most obese subjects. Although routine preoperative testing is not recommended in all, post operatively a high level of vigilance must be maintained for warning signs and symptoms. It is the duty of the primary surgeon and the nutritionists to counsel the patient to stick to a healthy diet and adequately supplement and monitor common vitamins and minerals. Early and appropriate intervention can prevent major disasters.

\section{Acknowledgements}

None.

\section{Conflict of interest}

The author declares no conflict of interest. 


\section{References}

1. Junshi Chen. An original discovery: selenium deficiency and Keshan disease (an endemic heart disease). Asia Pac JClin Nutr. 2012;21(3):320326.

2. Boldery R, Fielding G, Rafter T, et al. Nutritional deficiency of selenium secondary to weight loss (bariatric) surgery associated with lifethreatening cardiomyopathy. Heart Lung Circul. 2007;16(2):123-126.

3. Shankar P, Boylan M, Sriram K. Micronutrient deficiencies after bariatric surgery. Nutrition. 2010;26(11-12):1031-1037.

4. Xanthakos SA. Nutritional Deficiencies in Obesity and After Bariatric Surgery. Pediatr Clin North Am. 2009;56(5):1105-1121.

5. Weng TC, Chia-Hsuin Chang, Yaa-Hui Dong, et al. Anaemia and related nutrient deficiencies after Roux-en-Y Gastric Bypass surgery:a systematic review and meta-analysis. BMJ Open. 2015;5:e006964.
6. Gudzune KA, Huizinga MM, Chang HY, et al. Screening and diagnosis of micronutrient deficiencies before and after bariatric surgery. Obes Surg. 2013;23(10):1581-1589.

7. Ernst B, Thurnheer M, Schmid SM, et al. Evidence for the Necessity to Systematically Assess Micronutrient Status Prior to Bariatric Surgery. Obes Surg. 2009; 19:66-73.

8. Nett P, Borbély Y, Kröll D. Micronutrient Supplementation after Biliopancreatic Diversion with Duodenal Switch in the Long Term. Obes Surg. 2016;26(10):2469-2474.

9. Huseini M, Raza N. Selenium Deficiency Causing Cardiomyopathy in a Patient with Gastric Bypass Surgery. 2012.

10. Mechanick JI, Youdim A, Jones DB, et al. Clinical practice guidelines for perioperative nutritional, metabolic, and nonsurgical support of the bariatric surgery patient. Endo Pract. 2013;19(2):337-372. 